\title{
Association between Dietary Intake of Phytochemicals and hs-CRP in Healthy Women from Tehran: a Holistic Approach Using Dietary Phytochemical Index
}

\author{
Sareh Edalati $^{* 1}$, Beitolah Alipour ${ }^{2}$, Bahram Rashidkhani ${ }^{3}$ \\ 1-Student Resarch Committee, Faculty of Nutrition Sciences and Food Technology, Shahid Beheshti University of Medical Sciences, Tehran, Iran \\ 2- Faculty of Nutrition, Tabriz University of Medical Sciences, Tabriz, Iran \\ 3- Department of Community Nutrition, Faculty of Nutrition Sciences and Food Technology, Shahid Beheshti University of Medical Sciences, Tehran, \\ Iran
}

\section{A B S T R A C T}

Background and Objectives: Although the anti-inflammatory effects of phytochemicals have been supported by several in vitro studies, their biological actions from regular diets are mixed. This study aimed to use dietary phytochemical index (DPI), as a food-based framework to assess dietary phytochemical intake and to understand its association with hs-CRP in a sample of Iranian women. Evaluation of the relationship between hs-CRP and several anthropometric indexes were also assessed.

Materials and Methods: A total of 170 visibly healthy premenopausal women with the mean age of 35.2 were selected from health centers of Tehran via multistage cluster random sampling. The usual dietary intakes were assessed using a semi-quantitative food frequency questionnaire and dietary phytochemical intake was determined. General linear model was used to compare hs-CRP levels between tertile of DPI. Spearman correlation was used to assess the relationship between hs-CRP and the obesity indexes.

Results: The mean of DPI was 28.54. Significant spearman correlation coefficients were found between hs-CRP and WC (0.43), BMI (0.35), Waist to hip ratio (0.30), visceral adiposity index (0.28) (spearman's coefficient of correlation). No difference was found for hs-CRP concentration among different tertiles of dietary DPI, after adjustment for confounding factors including BMI, WC, and physical activity (P for trend=0.964).

Conclusions: The result of the present study showed that hs-CRP is associated with several central obesity indexes but no association was found between hs-CRP and dietary phytochemical index. Further studies using other biomarkers of inflammation and dietary assessment methods are needed to clarify the health effects of flavonoids in humans.

Keywords: Phytochemicals, Inflammation, Women

\section{Introduction}

Non-communicable diseases (NCD) including cardiovascular disease (CVD) is the most leading cause of death worldwide (1) including Iran (2). Beside conventional CVD risk factors, hs-CRP, a marker of inflammation, has been demonstrated to be an independent predictor of CVD $(3,4)$. Therefore, identification of dietary and lifestyle factors that may impact hs-CRP levels may have implication for prevention of CVD (5).

Although phytochemicals, chemical compounds produced by plants, are among dietary factors that have been proposed to be protective against CVD through suppressing inflammation in many in vitro and human trial studies $(6,7)$, the exact association of regular phytochemical content of human diet with clinical inflammation is not yet fully investigated. Previous studies have evaluated the relationship between dietary intake of phytochemicals and biomarkers of disease risk including inflammation, mostly focusing on separate food groups $(8,9)$ or specific classes of phytochemicals (10-13). As people consume a wide variety of foods, not isolated phytochemicals, adding a holistic approach which integrates all phytochemical components and takes 
into account the phytochemical and nutrients synergistic effects may be of more importance (1416). Furthermore, although the reductionist approach might unravel the mechanistic aspects of food and health relationships, using the holistic methods, besides having a reductionist look, could result in more relevant public health messages. It also can be used as a more practical tracking tool in public health research for monitoring diets and their health effects $(15,17-19)$.

Up to now, dietary phytochemical index (DPI), a priori food based dietary score has been developed to specially assess combined effects of all phytochemicals, and it is defined as the percent of dietary calories derived from foods rich in phytochemicals $(20,21)$. Among the existing studies that focus on the relationship between DPI and health outcomes, only one previous study has examined the association between inflammatory biomarkers and dietary PI which was conducted among a small sample of American population (22). This study aimed to use dietary PI as a food-based framework to assess phytochemical intake and to understand its association with hs-CRP in a sample of Iranian women. The relationship between hs-CRP and anthropometric indexes was assessed too.

\section{Materials and Methods}

Study population: The sample consisted of 170 visibly healthy premenopausal women (age range: 20 48) attending the health centers in Tehran. The multistage cluster random sampling was used to recruit these women. Shahid Beheshti University of Medical Sciences covers 3 main regions in Tehran (North, East and West). Each region (strata) has 21 health centers (clusters). Three health centers were randomly chosen from the East, 3 health centers from the West and 2 centers from the North of Tehran. The number of subjects in each health center was then calculated based on the proportion of coverage of each health center and then subjects were randomly selected from the sampling frame in each randomly selected center. For detection of the weakest correlation $(\mathrm{r}=0.3)$ with $\alpha$ error $=0.05$ and $\beta$ error $=0.1$, the total sample size required was $114(\mathrm{n}=114)$. However for improving the precision of the study, the sample size was increased to 170 subjects in our study (23)

Premenopausal is defined as being menstruated in a usual pattern in the last 3 months and not experiencing removal of the uterus and/or ovaries(24). Those who had a history of cancer or autoimmune, liver or renal dysfunctions, diabetes, hypertension, hypothyroidism, hyperthyroidism, infection during the past six months, or being pregnant, lactating, and experiencing menopause were excluded from the study. Other exclusion criteria were as follows: use of any medications and supplements known to influence the variables studied (lipid-lowering drugs, contraceptive drugs, herbal and antioxidant Supplements, probiotic supplements, antibiotics, hormone replacement therapy), a restrictive diet or any change in dietary patterns (12). The participants with elevated hs-CRP above $10 \mathrm{mg} / \mathrm{L}$ were excluded, possibly indicating an acute inflammation (25), however no one had hs-CRP above $10 \mathrm{mg} / \mathrm{L}$.

After considering the exclusion criteria, Among 190 people who were invited, 170 of them agreed to participate in the study and written consent was given by each subject. The procedures of this study were approved by the medical Ethics Committee of Tabriz University of medical sciences as well as Shahid Beheshti University of medical sciences.

Assessment of dietary intake: Usual Dietary intake was assessed using a 168 item validated semi quantitative food frequency questionnaire tailored specifically for the Iranian population. During an face to face interview, each participant was asked to report how often, on average, he/she has eaten each food item on a daily, weekly and monthly basis over the previous year (26). Portion sizes of consumed foods were converted to grams by using household measures (27). Energy intakes were computed using the Iranian food composition table (FCT) (28) and US Department of Agriculture's (USDA FCT) using N4 software. The USDA food composition data included in the Nutritionist 4 software (First Databank; Hearst, San Bruno, CA, USA) was used to calculate the energy and nutrient content of foods. For Iranian food items which are not included in Nutritionist 4 , such as kashk (a dairy food), the IFCT was used.

DPI was calculated using the formula DPI= [daily energy derived from phytochemical-rich foods $\mathrm{kcal} /$ total daily energy intake kcal) $\times 100$ ]. Dietary phytochemical index (DPI) included different phytochemical rich food groups including whole grains (whole wheat bread, oat and bulgur), fruits (red, yellow and orange fruits), vegetables (dark green vegetables, red/orange vegetables, starchy vegetables and other vegetables), natural fruit and vegetable 
juices, tomato sauces, soy products (soy bean), nuts (peanut, almond, walnut, pistachio and hazelnut), legumes (lentil, beans, chickpea, split bean, mung bean and vicia faba),olive and olive oil. Potato was not considered as a vegetable group due to its low phytochemical content(20).

Biochemical measurement: Blood samples were drawn after 12-14 hours of fasting and then centrifuged for $10 \mathrm{~min}$ at $3,000 \mathrm{rpm}$ to separate serum. Serum aliquots were stored at $-70{ }^{\circ} \mathrm{C}$ until the day of analysis. Hs-CRP was estimated by ELISA [Diagnostics Biochem Canada Inc (DBC), Ontario Canada] in the research laboratory of Endocrine research Center of Shahid Beheshti Medical University. Serum total cholesterol (TC), triglyceride (TG), and high-density lipoprotein cholesterol (HDLC), were assayed using commercial kits (Pars Azmoon Inc, Tehran, Iran) by enzymatic methods and a Selectra 2 auto-analyzer (Vital Scientific, Spankeren, Netherlands).

Assessment of other variables: Physical activity was determined using a self-reported-based questionnaire (29) and expressed as metabolic equivalent hours per day (MET-h/day) (30). Height was measured using a stadiometer (Seca, model 206, Hamburg, Germany) fixed to the wall, without shoes and headdress using the Frankfort Plane Technique (31).Weight was determined to the nearest $0.1 \mathrm{~kg}$ using a digital scale (Seca 707, Hamburg, Germany), while the participant had light clothing and were barefoot. Body mass index (BMI) was calculated by dividing weight by height squared $\left(\mathrm{kg} / \mathrm{m}^{2}\right)$. Waist and hip circumferences were measured using a Seca 201 measuring tape according to the World Health Organization (WHO) protocol (32). The waist-to-hip ratio (WHR) was calculated as WC divided by hip circumference (both in $\mathrm{cm}$ ). The visceral adiposity index was calculated using the formula for Females: [VAI=(WC/ $36.58+$ $(1.89 \times \mathrm{BMI}) \times(\mathrm{TG} / 0.81) \times(1.52 / \mathrm{HDL})](33)$.

Statistical analysis: Statistical analysis was performed with SPSS 21 for Windows (SPSS Inc., Chicago, IL). The normality of data was tested by one-sample Kolmogorov-Smirnov test. PI index was assigned as tertile intakes, based on their 33th, 66th, 99th values, and general linear model (GLM) was used to compare the mean $( \pm \mathrm{SE})$ values of hs-CRP (dependent variables) and phytochemical rich foods across groups (fixed factor) after adjustments for confounding factors, including BMI, WC, and physical activity. Spearman correlation was used for the association between hs-CRP and central obesity indexes.

\section{Results}

The characteristics of the study participants across tertiles categories of PI are shown in Table 1.

Individuals in the highest tertile of PI were significantly older. No significant differences were observed in physical activity, BMI, WC, waist to hip ratio, VAI and energy intake within groups investigated. Hs-CRP significantly correlated with age, BMI, WC, Waist to hip ratio and visceral adiposity index (Table 2).

Table 3 compares the mean intake of Phytochemical-rich foods across tertiles of PI after adjusting for age. There was an increasing trend in dietary intakes of fruits, vegetables, whole grains, olive and olive oil across tertile categories of dietary PI $(p \leq 0.001)$.

The relation between PI and plasma hs-CRP concentrations is shown in Table 4. No difference was found for hs-CRP concentration among different tertiles of dietary PI, after adjustment for confounding factors including age, WC and physical activity.

Table 1. Baseline characteristics of participants across tertiles of energy adjusted flavonoid intake

\begin{tabular}{|c|c|c|c|c|}
\hline \multirow[b]{2}{*}{ Variables } & \multicolumn{4}{|c|}{ Tertiles of dietary phytochemical index } \\
\hline & $\begin{array}{l}\text { First tertile } \\
\qquad(\mathrm{n}=56) \\
0-23.76\end{array}$ & $\begin{array}{c}\text { Second tertile } \\
(\mathrm{n}=75) \\
23.76-31.2\end{array}$ & $\begin{array}{c}\text { Third tertile } \\
\quad(n=57) \\
>31.21\end{array}$ & $\mathrm{P}$ for trend \\
\hline Age (years) & $32.60 \pm 0.83^{*}$ & $36.21 \pm 0.82$ & $36.94 \pm 0.82$ & $<0.001$ \\
\hline Physical activity (Met-hour/day) & $44.72 \pm 0.45$ & $44.55 \pm 0.45$ & $43.70 \pm 0.45$ & 0.32 \\
\hline Body mass index (kg/m2) & $27.93 \pm 0.61$ & $28.01 \pm 0.59$ & $28.11 \pm 0.59$ & 0.86 \\
\hline Waist circumference $(\mathrm{cm})$ & $87.82 \pm 1.24$ & $90.14 \pm 1.20$ & $88.61 \pm 1.21$ & 0.39 \\
\hline Waist to hip ratio & $0.83 \pm 0.07$ & $0.84 \pm 0.07$ & $0.84 \pm 0.07$ & 0.69 \\
\hline Visceral adiposity index & $367.90 \pm 37.84$ & $419.00 \pm 36.56$ & $361.75 \pm 36.85$ & 0.47 \\
\hline Total energy intake (kcal/day) & $2290 \pm 72.98$ & $2203 \pm 72.34$ & $2269 \pm 72.34$ & 0.84 \\
\hline
\end{tabular}

"Data are presented as mean $\pm \mathrm{SE}$

$P$-value for linear trend over tertiles of intake 
Table 2. Correlation of hs-CRP, anthropometric indexes and biochemical biomarkers in apparently healthy woman ${ }^{1}$

\begin{tabular}{lcc}
\hline \multicolumn{1}{c}{ Variables } & Correlation coefficient & P value \\
\hline Age (years) & 0.17 & 0.02 \\
Physical activity(Met-hour/day) & -0.03 & 0.652 \\
Waist circumference (cm) & 0.43 & $<0.001$ \\
Body mass index $\left(\mathbf{k g} / \mathbf{m}^{2)}\right.$ & 0.35 & $<0.001$ \\
Waist / hip ratio & 0.30 & $<0.001$ \\
Visceral adiposity index & 0.28 & $<0.001$ \\
\hline 1. Spearman test & &
\end{tabular}

Table 3. Dietary intake of phytochemical rich food groups to tertiles of dietary phytochemical index

\begin{tabular}{|c|c|c|c|c|}
\hline \multirow[b]{2}{*}{ Variables } & \multicolumn{4}{|c|}{ Tertiles of dietary phytochemical index } \\
\hline & $\begin{array}{l}\text { First tertile } \\
\quad(n=56)\end{array}$ & $\begin{array}{l}\text { Second tertile } \\
(n=75)\end{array}$ & $\begin{array}{l}\text { Third tertile } \\
(\mathrm{n}=57)\end{array}$ & P for trend ${ }^{1}$ \\
\hline $\begin{array}{l}\text { Dietary phytochemical index } \\
\text { (percent) }\end{array}$ & $19.14 \pm 0.58^{*}$ & $27.15 \pm 0.57$ & $39.17 \pm 0.57$ & $<0.001$ \\
\hline Carbohydrate (\% of total energy) & $56.03 \pm 0.74$ & $58.51 \pm 0.72$ & $58.98 \pm 0.72$ & 0.014 \\
\hline Total fat (\% of total energy) & $32.605 \pm 0.77$ & $30.283 \pm 0.75$ & $30.08 \pm 0.75$ & 0.038 \\
\hline Total protein (\% of total energy) & $13.419 \pm 0.29$ & $13.65 \pm 0.28$ & $14.20 \pm 0.28$ & 0.149 \\
\hline Fruits (gr/day) & $284.033 \pm 25.537$ & $425.937 \pm 25.312$ & $554.177 \pm 25.312$ & $<0.001$ \\
\hline Vegetables (gr/day) & $271.485 \pm 20.797$ & $350.031 \pm 20.613$ & $378.155 \pm 20.613$ & $<0.001$ \\
\hline whole grain(gr/day) & $22.7 \pm 6.7$ & $30.3 \pm 6.6$ & $81.1 \pm 6.6$ & $<0.001$ \\
\hline Olive and olive oil(gr/day) & $1.2 \pm 0.5$ & $2.3 \pm 0.5$ & $3.3 \pm 0.5$ & 0.004 \\
\hline Cereals (gr/day) & $38 / 0 \pm 4.3$ & $53.2 \pm 4.3$ & $49.1 \pm 4.3$ & 0.081 \\
\hline Soy (gr/day) & $3.1 \pm 0.6$ & $3.7 \pm 0.6$ & $3.9 \pm 0.6$ & 0.412 \\
\hline Nuts (gr/day) & $37.8 \pm 3.3$ & $10.0 \pm 3.3$ & $8.2 \pm 3.3$ & 0.971 \\
\hline
\end{tabular}

"Data are presented as mean \pm SE

Glm analysis, independent variable: tertiles of DPI, dependent variable: dietary intakes, $P$-value for linear trend over tertiles of intake, adjusted for Age

Table 4. General linear model of the association between hs-CRP and dietary phytochemical intake measured by the dietary phytochemical index (DPI).

\begin{tabular}{ccccc}
\hline variable & \multicolumn{4}{c}{ Tertiles of dietary phytochemical index } \\
\cline { 2 - 4 } & $\begin{array}{c}\text { First tertile } \\
(\mathrm{n}=56)\end{array}$ & $\begin{array}{c}\text { Second tertile } \\
(\mathrm{n}=57)\end{array}$ & $\begin{array}{c}\text { Third tertile } \\
(\mathrm{n}=57)\end{array}$ & P for trend $^{1}$ \\
\hline $\begin{array}{c}\text { hs-CRP } \\
(\mathbf{m g} / \mathbf{L})\end{array}$ & $2.77(2.02-3.52)^{*}$ & $2.51(1.79-3.24)$ & $2.81(2.08-3.53)$ & 0.964 \\
\hline Glm analyses after controlling for age, BMI and waist circumference. Independent variable: tertiles of DPI, dependent variable: $\log$ hs-CRP $*$ mean $(95 \mathrm{CI})$
\end{tabular}

\section{Discussion}

Despite the higher intakes of some kinds of phytochemicals rich groups in the highest tertile of DPI in comparison with lower tertile, no significant differences in hs-CRP levels were seen across tertiles of dietary phytochemical index. The results of the present study is consistent with the findings of Vincet et al, which showed no significant relationship between dietary phytochemical index and hs-CRP in 54 American individuals aged 18-30 (22). As tea was the main contributor of total flavonoids intake with the mean intake of 3.12 (cup/day) in our population (data not published), not taking into account noncaloric herbal teas in DPI-might lead to the null association. Mixed results on the anti-inflammatory activity of phytochemicals in humans may also be due to their low absorptions. Furthermore, differences in gut microbiota composition and the food matrix may affect phytochemicals bioavailability and eventually its efficacy $(34,35)$. Therefore, more studies on dietary intake of phytochemicals and its health effects among different ethnic groups needs to be carried out. Applying different measures for estimations of phytochemical intakes focusing on separate food groups (8) or specific classes of phytochemicals using different databases (10-13) may link to different results $(16,36)$. In this regard, studies aiming to explore biochemical biomarkers reflecting dietary intake of phytochemicals are required and also to validate dietary driven data might be helpful (37).

The anti-inflammatory effects of phytochemicals in human diet may be different from those gained from experimental studies due to the use of nonphysiologically relevant forms or concentrations of phytochemicals (38). Low serum levels and chemical 
modifications of dietary phytochemicals in vivo may affect their biological activities (39). It seems that the biological health benefits of phytochemicals is mostly through their prebiotics acts in humans (40).

The secondary aim of the present study was to evaluate the relationship between hs-CRP and anthropometric variables. A positive association between hs-CRP and visceral obesity indices including VAI are in line with some previous studies $(41,42)$. The positive association between obesity and hs-CRP highlights the need for policy making practices to prevent obesity in Iranian population.

Potential confounding factors that assumed to affect VAI or hs-CRP were controlled as excluding criteria. When interpreting the results of the present study, inherent limitations within the cross- sectional design of the study as well as the measurement errors of dietary data (43) needed to be considered. To reduce clinical inflammation via dietary modifications, more research is suggested to develop dietary models considering nutrition effects, cultural acceptance and the affordability considerations (16, 44).

\section{Conclusion}

The result of the present study showed that hsCRP is associated with several indexes of central obesity but no association was found between hs-CRP and dietary phytochemical index. More studies using other biomarkers of inflammation and dietary assessment methods are needed to clarify the conflicting health effects of flavonoids in humans.

\section{Financial disclosure}

We confirm that this manuscript did not lead to any conflict of interests regarding the publication.

\section{Funding/Support}

This research project was financially supported by Tabriz Medical University.

\section{References}

1. Mozaffarian D, Benjamin EJ, Go AS, Arnett DK, Blaha MJ, Cushman M, et al. Executive summary: heart disease and stroke Statistics-2016 update: a report from the American Heart Association. Circulation. 2016;133(4):447-54.

2. Saadat S, Yousefifard M, Asady H, Jafari AM, Fayaz M, Hosseini M. The most important causes of death in Iranian population; a Retrospective Cohort Study. Emergency. 2015;3(1):16.

3. Ridker PM. Clinical application of C-reactive protein for cardiovascular disease detection and prevention. Circulation. 2003;107(3):363-9.
4. Salazar J, Martínez MS, Chávez M, Toledo A, Añez R, Torres Y, et al. C-reactive protein: clinical and epidemiological perspectives. Cardiol Res Pract. 2014;2014, Article ID 605810, 10 pages

5. Esposito K, Giugliano D. Diet and inflammation: a link to metabolic and cardiovascular diseases. Eur Heart J. 2005;27(1):15-20.

6. Gencoglu H, Orhan C, Sahin K. Phytochemical Therapies in Vascular Functioning: A Molecular Approach. Curr Vasc Pharmacol. 2017;15(4):327-38.

7. Rotelli AE, Guardia T, Juárez AO, De la Rocha NE, Pelzer LE. Comparative study of flavonoids in experimental models of inflammation. Pharmacol Res. 2003;48(6):601-6.

8. Jiang Y, Wu S-H, Shu X-O, Xiang Y-B, Ji B-T, Milne $\mathrm{GL}$, et al. Cruciferous vegetable intake is inversely correlated with circulating levels of proinflammatory markers in women. J Acad Nutr Diet. 2014;114(5):7008. e2.

9. Haghighatdoost F, Bellissimo N, de Zepetnek JOT, Rouhani MH. Association of vegetarian diet with inflammatory biomarkers: a systematic review and meta-analysis of observational studies. Public Health Nutr. 2017;20(15):2713-21.

10. Cassidy A, Rogers G, Peterson JJ, Dwyer JT, Lin H, Jacques PF. Higher dietary anthocyanin and flavonol intakes are associated with anti-inflammatory effects in a population of US adults. Am J Clin Nutr. 2015;102(1):172-81.

11. Jennings A, Welch AA, Spector T, Macgregor A, Cassidy A. Intakes of anthocyanins and flavones are associated with biomarkers of insulin resistance and inflammation in women. J Nutr. 2014;144(2):202-8.

12. Alipour B, Rashidkhani B, Edalati S. Dietary flavonoid intake, total antioxidant capacity and lipid oxidative damage: A cross-sectional study of Iranian women. Nutrition. 2016;32(5):566-72.

13. Vitale M, Vaccaro O, Masulli M, Bonora E, Del Prato $\mathrm{S}$, Giorda $\mathrm{CB}$, et al. Polyphenol intake and cardiovascular risk factors in a population with type 2 diabetes: The TOSCA. IT study. Clin Nutr. 2017;36(6):1686-92.

14. Fardet A. Towards a more Holistic Vision of Human Nutrition to Prevent from Diet-Related Chronic diseases: the reductionist drift. Int J Food Sci Nutr Diet. 2016;5:1-2.

15. Fardet A, Rock E. The search for a new paradigm to study micronutrient and phytochemical bioavailability: from reductionism to holism. Med Hypotheses. 2014;82(2):181-6.

16.Tapsell LC, Neale EP, Satija A, Hu FB. Foods, nutrients, and dietary patterns: interconnections and implications for dietary guidelines. Advances in Nutrition: . Advances in Nutrition. 2016;7(3):445-54.

17. Messina M, Lampe JW, Birt DF, Appel LJ. Reductionism and the narrowing nutrition perspective: time for reevaluation and emphasis on food synergy. Int J Food Sci Nutr Diet. 2001;101(12):1416. 
18. Fardet A, Rock E. Toward a new philosophy of preventive nutrition: from a reductionist to a holistic paradigm to improve nutritional recommendations. Advances in Nutrition: Adv Nutr. 2014;5(4):430-46.

19. Jacobs Jr DR, Temple NJ. Food synergy: a paradigm shift in nutrition science. Nutritional health: Springer; 2012. p. 311-22.

20. McCarty MF. Proposal for a dietary "phytochemical index". Med Hypotheses. 2004;63(5):813-7.

21. Bahadoran Z, Golzarand M, Mirmiran P, Saadati N, Azizi F. The association of dietary phytochemical index and cardiometabolic risk factors in adults: Tehran Lipid and Glucose Study. J Hum Nutr Diet. 2013;26(s1):14553.

22. Vincent HK, Bourguignon CM, Taylor AG. Relationship of the dietary phytochemical index to weight gain, oxidative stress and inflammation in overweight young adults. J Hum Nutr Diet. 2010;23(1):20-9.

23. Norman G, Streiner D. Biostatistics: the bare essentials. Hamilton, Ontario: BC Decker. Inc Google Scholar. 2000:106.

24. McKinlay SM, Brambilla DJ, Posner JG. The normal menopause transition. Maturitas. 1992;14(2):103-15.

25.Zwirska-Korczala K, Konturek S, Sodowski M, Wylezol M, Kuka D, Sowa P, et al. Basal and Postprandial Plasma Levels of Pyy, Ghrelin. J Physiol Pharmacol. 2007;58:13-35.

26. Esfahani FH, Asghari G, Mirmiran P, Azizi F. Reproducibility and relative validity of food group intake in a food frequency questionnaire developed for the Tehran Lipid and Glucose Study. J Epidemiol. 2010;20(2):150-8.

27. Ghaffarpour M H-RA, Kianfar H. The manual for household measures, cooking yields factors and edible portion of foods (In Persian). Tehran: Keshavarzi Press; 1999.

28. Azar M, Sarkisian E. Food composition table of Iran: National Nutrition and Food Research Institute. Shaheed Beheshti University, Tehran. 1980.

29. Aadahl M, Jørgensen T. Validation of a new self-report instrument for measuring physical activity. Med Sci Sports Exerc. 2003;35(7):1196-202.

30. Ainsworth BE, Haskell WL, Whitt MC, Irwin ML, Swartz AM, Strath SJ, et al. Compendium of physical activities: an update of activity codes and MET intensities. Med Sci Sports Exerc. 2000;32(9; SUPP/1):S498-S504.

31. de Onis M, Onyango AW, Van den Broeck J, Chumlea WC, Martorell R. Measurement and standardization protocols for anthropometry used in the construction of a new international growth reference. Food Nutr Bull. 2004;25(1 Suppl):S27-36.

32. Organization WH. Measuring obesity-classification and description of anthropometric data. Report on a WHO consultation of the epidemiology of obesity. Warsaw 21-23 October 1987. Copenhagen: WHO, 1989. Nutrition Unit document, EUR/ICP/NUT. $1987 ; 123$.

33. Amato MC, Giordano C. Visceral adiposity index: an indicator of adipose tissue dysfunction. . Int $\mathbf{J}$ Endocrinol. 2014;2014.

34. Cassidy A, Minihane A-M. The role of metabolism (and the microbiome) in defining the clinical efficacy of dietary flavonoids. Am J Clin Nutr. 2017;105(1):10-22.

35. Lampe JW. Interindividual differences in response to plant-based diets: implications for cancer risk. The Am J Clin Nutr. 2009;89(5):1553S-7S.

36. Mossavar-Rahmani Y. Reducing measurement error in nutrition assessment: potential research implications for Iran. . Nutr Food Sci Res. 2017;4(1):3-10.

37. Jenab M, Slimani N, Bictash M, Ferrari P, Bingham SA. Biomarkers in nutritional epidemiology: applications, needs and new horizons. Hum Genet. 2009; 125(5-6):507-25.

38. Kay CD. The future of flavonoid research. Br J Nutr. 2010;104(S3):S91-S5.

39. Lotito SB, Zhang W-J, Yang CS, Crozier A, Frei B. Metabolic conversion of dietary flavonoids alters their anti-inflammatory and antioxidant properties. Free Radic Biol Med. 2011;51(2):454-63.

40. Williamson G, Clifford MN. Colonic metabolites of berry polyphenols: the missing link to biological activity? Br J Nutr.2010;104(S3):S48-S66.

41. Brooks GC, Blaha MJ, Blumenthal RS. Relation of Creactive protein to abdominal adiposity. Am J Cardiol. 2010;106(1):56-61.

42. Al-Daghri NM, Al-Attas OS, Alokail M, Alkharfy K, Wani K, Amer OE, et al. Does visceral adiposity index signify early metabolic risk in children and adolescents?: association with insulin resistance, adipokines, and subclinical inflammation. Pediatr Res. 2013;75(3):459-63.

43. Mendez MA. Invited commentary: dietary misreporting as a potential source of bias in diet-disease associations: future directions in nutritional epidemiology research. Am J Epidemiol.2015;181(4):234-6.

44. Drewnowski A. New metrics of affordable nutrition: which vegetables provide most nutrients for least cost? J Acad Nutr Diet. 2013;113(9):1182-7. 